current work in the authors' laboratory is also directed at this goal.

\section{REFERENCES}

Craik, F. I. M. A "levels of analysis" view of memory. In P. Pliner, L. Kramers, and T. M. Alloway (Eds.), Communication and affect: Language and thought. New York: Academic Press, 1973.

Craik, F. I. M., \& Lockhart, R. S. Levels of processing: A framework for memory research. Journal of Verbal Learning and Verbal Behavior, 1972, 11, 671-684.

Craik, F. I. M., \& Watkins, M. J. The role of rehearsal in short-term memory. Journal of Verbal Learning and Verbal Behavior, 1973, 12, 599-607.

Gardiner, J. M. Levels of processing in word recognition and subsequent free recall. Journal of Experimental Psychology, $1974,102,488-493$.
Hyde, T. S. Differential effects of effort and type of orienting task on recall and organization of highly associated words Journal of Experimental Psychology, 1973, 79, 111-113.

Hyde, T. S., \& Jenkins, J. J. Differential effects of incidental tasks on the organization of highly associated words. Journal of Experimental Psychology, 1969, 82, 472-481.

Jacoby, L., \& Bartz, W. H. Rehearsal and transfer to LTM. Journal of Verbal Learning and Verbal Behavior, 1972, 11, 561-566.

Kucera, H., \& Francis, W. M. Computational analysis of present-day American English. Providence: Brown University Press, 1967.

Meunier, G. F., Kesttner, J., Meunier, J. A., \& Ritz, D. Overt rehearsal and transfer to long-term memory. Journal of Experimental Psychology, 1974, 102, 913-914.

Shiffin, R. M. Information persistence in short-term memory. Journal of Experimental Psychology, 1973, 100, 39-49.

(Received for publication September 9, 1974.)

\title{
Determination of stimuli "neutral" with respect to generalization from horizontal-vertical stripes discrimination*
}

\author{
DAVID A. STEVENS \\ Clark University, Worcester, Massachusetts 01610
}

\begin{abstract}
To determine stimuli with which there would be no differential generalization when used in transfer tests, 10 rats learned a successive vertical-horizontal stripe discrimination and were then tested for generaliztion with the following stimuli: 4-cell checkerboard, lattice, and eight sets of stripes ranging in slope from $10 \mathrm{deg}$ to $80 \mathrm{deg}$. There was no differential generalization with the checkerboard, but there was generalization from horizontal stripes to the lattice. Interpolation from responses to stripes of 40-deg and 50-deg slopes indicated that with a slope of 42.9 deg there would be equal generalization from the horizontal and vertical standards.
\end{abstract}

Several techniques used to investigate discrimination learning involve transfer tests which require no differential stimulus generalization between stimuli used for training and those used for testing. For example, this characteristic defines the difference between reversal and

*The technical assistance of James W. Case is gratefully acknowledged. This study was supported by a grant from the Clark University Graduate Science Education Fund. nonreversal shift tests. In the former, complete stimulus generalization is obtained by using the same stimuli in training and test phases (acquisition and reversal), while in the latter, stimulus generalization is avoided by deliberately employing new stimuli for the test phase which differ along a different dimension than do the training stimuli. Another example is replaced stimulus tests. With these tests either $\mathrm{S}+$ or $\mathrm{S}$ - replaced by a new 
stimulus and $\mathrm{S}-$ or $\mathrm{S}+$, respectively, remains unchanged. Differential test performance is taken as evidence for differences in the strengths of associations between the retained stimuli and responses elicited by them (Mandler, 1968; Stevens, \& Fechter, 1968, for example). Should there be some stimulus generalization from one or both of the training stimuli to the new, test stimulus, a systematic bias would result.

Counterbalancing is usually employed to control for this bias. While this controls for additive effects of generalization if generalization is equal from the two training stimuli, it is not a satisfactory control if there is differential generalization from the two training stimuli to the new test stimulus. In this latter case, the error variance so produced could mask small transfer effects.

Despite the importance of controlling for differential generalization, empirical determinations of appropriate test stimuli are not usually done. Rather, rational criteria are used. The validity of this procedure is questionable, for generalization can in fact occur where logically it should not. For example, Law (1954) showed that homogeneous white stimuli generalize more to vertically striped stimuli than to horizontally striped stimuli, and, conversely, homogeneous black stimuli generalize more to horizontally than to vertically striped stimuli. Moreover, the generalization from black to horizontal stripes is more pronounced than from white to vertical stripes.

Since ongoing research in our laboratory involves giving replaced stimulus tests following acquisition of a horizontal vs vertical stripe discrimination, the present work was done to determine stimuli that would be appropriate for those tests. Two stimuli, selected on logical grounds, were tested for differential generalization. These two stimuli were a 4-cell checkerboard (previously used by Stevens \& Fechter, 1968) and a lattice consisting of overlying horizontal and vertical black stripes. These both had equal horizontal and vertical contours and like the striped stimuli; the checkerboard, but not the lattice, had equal areas of black and white. Simultaneously, a psychophysical bisection of the horizontal-vertical dimension was obtained using striped stimuli of varying slope. Such a bisection defines a stimulus having equal stimulus generalization from the horizontal and vertical standards.

\section{METHOD}

\section{Subjects}

The Ss were 10 male rats of the Charles River CD strain, about 90 days old.

\footnotetext{
Apparatus

The apparatus was a discrimination box similar to that described by McGaugh and Thomson (1962, Fig. 1). It was trapezoidal in shape, 27 in. long, 12 in. wide at one end and 6 in. wide at the other end, with a grid floor. A Plexiglas door 7 in. from the narrow end provided a startbox. Two openings, 3 in.
}

square and 6 in. apart were located in the wall at the wide end. A 3-in. partition separated the openings. Swinging doors, faced with stimulus cards, were located behind these openings. One door opened into a goalbox and the other was locked shut. In the goalbox was a small cylinder which, on reinforced trials, contained $0.2 \mathrm{ml}$ water. The entire apparatus was painted medium gray. To provide an objective determination of errors (responses to the incorrect door) two microammeters were used. For each meter, one pole was wired to one of the grid sections between the end of the partition and a door, and the other pole was wired in series with $220 \mathrm{~K} \mathrm{ohm}$ resistor, a $6-\mathrm{V}$ dry cell and remainder of the grid. When a $S$ touched the floor in front of either of the doors, it was indicated by deflection of the needle of the appropriate meter. The current passed (about 5 ua) was not aversive and was probably subliminal.

Thirteen pairs of stimulus cards were used. Homogeneous medium gray stimuli were used for pretraining. For acquisition training, the stimuli were pairs of horizontal and vertical alternating black and white $1 / 2$-in. stripes. For the generalization tests, the stimuli were pairs of black and white 4-cell checkerboards, lattices of three 1/2-in. black horizontal and vertical stripes overlaid on a white ground, and eight pairs of stimuli having alternating black and white $1 / 2$-in. stripes ranging in slope from $10 \mathrm{deg}$ to $80 \mathrm{deg}$ in $10-\mathrm{deg}$ increments.

\section{Procedure}

The Ss were gentled and adapted to a 23-h 50-min water deprivation schedule. They were then shaped to leave the startbox, push open a gray stimulus door and enter the goalbox. A $0.2-\mathrm{ml}$ water reinforcer was used in this and subsequent phases of the experiment. A gray block was placed in front of the locked door, forcing the Ss to respond to the unlocked one. The location of the unlocked door was determined by Gellerman sequences throughout the experiment. Following pretraining, all Ss were trained on a horizontal stripes (0-deg slope) vs vertical stripes (90-deg slope) successive discrimination. On any trial either two horizontally striped stimuli or two vertically striped stimuli were visible. For six Ss the left-hand stimulus was correct when horizontal stripes were present, and the right-hand stimulus was correct when the vertical stripes were present. The opposite relationship held for the remaining four Ss. Fifteen trials a day were given until a criterion of 27 correct responses within 2 successive days was met. The $S$ then began the test phase. It continued to receive 15 trials a day. Of these, 10 were indentical to acquisition trials. The remaining five were test trials which employed the 10 sets of test stimuli (eight sets of striped stimuli ranging in slope from $10 \mathrm{deg}$ through $80 \mathrm{deg}$, the 4-cell checkerboard, and the lattice). These sets were presented ten times each in sequences determined by a $10 \times 10$ Latin square. The sequence of training and test trials within a day was determined randomly with the condition that at least one training trial fall between test trials. All training trials were reinforced; test trials were alternately reinforced or not.

\section{RESULTS AND DISCUSSION}

The mean percent responses appropriate for the horizontal striped stimuli made for each of the 10 test stimuli are shown in Fig. 1 together with the 95\% (two-tailed) confidence limits. As can be seen in that figure, the checkerboard stimulus produced responses almost evenly divided between those appropriate for horizontal and vertical stripes (49\%:51\%). The confidence limits for percentage of "horizontal" responses were 60.9 and 37.1. On the other hand, responses to the lattice were clearly biased, with $59 \%$ of 


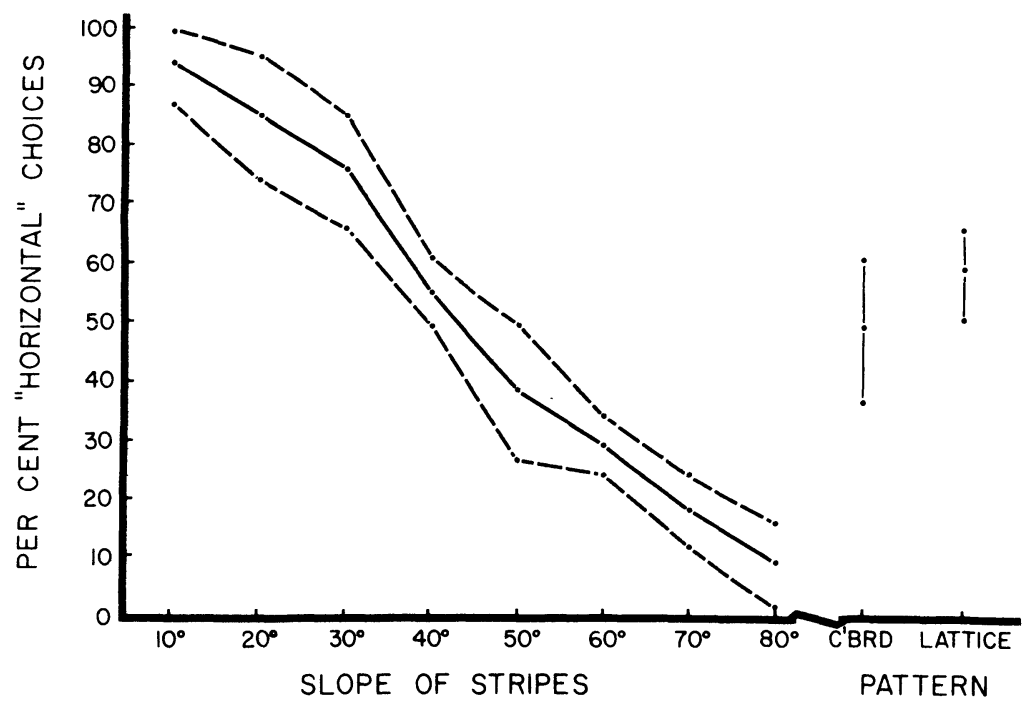

Fig. 1. Mean percentage of responses to test stimuli that were appropriate for the horizontally striped training stimulus, with 95\% confidence limits shown about each point. the responses to that stimulus being appropriate for the horizontal stimuli. The confidence limits were $66.9 \%$ and $51.1 \%$. Thus, responses to the lattice were significantly different from the $50 \%$ level expected from a neutral stimulus.

To determine the slope of the striped stimuli that would produce responses appropriate for both horizontal and vertical stimuli with equal frequency, two computations were done. First, that specific slope which would produce responses appropriate for the horizontal stimuli on half the trials was determined by interpolation using the results from the 40-deg and 50 -deg stimuli. The interpolation produced a slope of $42.9 \mathrm{deg}$. Second, the range of slopes that would produce responses appropriate for horizontal stimuli to a degree not significantly different from chance was determined. This was done by computing tile intercept of the higher and lower confidence limits with the line representing a chance level of performance. The results of those interpolations showed confidence limits of $39.4 \mathrm{deg}$ and $48.8 \mathrm{deg}$.

The performance found with the 50-deg slope was significantly different from chance while that found with the 40-deg slope was not. Yet, both of these slopes differ by $40 \mathrm{deg}$ from the vertically and horizontally striped standards, respectively. This suggested that rats analyze verticality and horizontality with differential efficiency. Therefore, the data from the eight striped test stimuli were also analyzed to determine if there was a significant difference in performance as a function of deviation of slope away from the vertical standard as opposed to deviation away from horizontal standard. A two-way ANOVA for repeated measures was done to compare performance as the stimuli changes in 10-deg increments away from horizontal (10 deg through 40 deg slopes) with performance as the stimuli changed away from vertical in 10-deg increments ( 80 deg through $50 \mathrm{deg}$ slopes). The analysis indicated a main effect for slope deviation in general $(F=29.61$, df $=3 / 27$, $p<.001$ ) but no significant main effect for horizontal vs vertical standards $(\mathrm{F}=0.06)$ or the interaction $(\mathrm{F}=$ 1.66) was found. Thus there is no evidence for differential efficiency of horizontal vs vertical slope detection.

The results of this study indicate tinat there is no differential stimulus generalization from horizontally and vertically striped patterns to a 4-cell checkerboard pattern. The psychophysical bisection indicated that stripes with a slope of 42.9 deg define a stimulus having equal generalization from vertically and horizontally striped standards. Therefore, either of these stimuli are appropriate for experiments in which a lack of differential generalization from horizontally and vertically stimuli is essential.

The lattice composed of overlying horizontal and vertical stripes was judged as more horizontal than vertical by the Ss in this study. This bias was unexpected inasmuch as the lattice had equal horizontal and vertical contours. The greater generalization from the horizontal stimuli may lie in the fact that the ratio of black and white areas was $75: 25$. Thus, the higher proportion of "horizontal" responses to the lattice is consistent with Law's (1954) finding of a preference for horizontal stimuli by rats having been trained to respond to black stimuli.

\section{REFERENCES}

Law, O. T. Preference in the rat for vertical or horizontal stripes after training on a white-black discrimination. American Journal of Psychology, 1954, 67, 714-716.

Mandler, J. M. Overtraining and the use of positive and negative stimuli in reversal and transfer. Journal of Comparative \& Physiological Psychology, 1968, 66, 110-115.

McGaugh, J. L., \& Thomson, C. W. Facilitation of simultaneous discrimination with strychnine sulphate Psychopharm acologia, 1962,3, 166-172.

Stevens, D. A., \& Fechter, L. D. Relative strengths of approach and avoidance tendencies in discrimination learning of rats trained under two types of reinforcement. Journal of Experimental Psychology, 1968, 76, 489-491. 